\title{
Influence of nanoporesize on platelet adhesion and activation
}

\author{
Natalia Ferraz $\cdot$ Jan Carlsson $\cdot$ Jaan Hong $\cdot$ \\ Marjam Karlsson Ott
}

Received: 28 January 2008/Accepted: 3 April 2008/Published online: 15 April 2008

(C) Springer Science+Business Media, LLC 2008

\begin{abstract}
In this study we have evaluated the influence of biomaterial nano-topography on platelet adhesion and activation. Nano-porous alumina membranes with pore diameters of 20 and $200 \mathrm{~nm}$ were incubated with whole blood and platelet rich plasma. Platelet number, adhesion and activation were determined by using a coulter hematology analyzer, scanning electron microscopy, immunocytochemical staining in combination with light microscopy and by enzyme immunoassay. Special attention was paid to cell morphology, microparticle generation, Pselectin expression and $\beta$-TG production. Very few platelets were found on the $200 \mathrm{~nm}$ alumina as compared to the $20 \mathrm{~nm}$ membrane. The platelets found on the $20 \mathrm{~nm}$ membrane showed signs of activation such as spread morphology and protruding filipodia as well as P-selectin expression. However no microparticles were detected on this surface. Despite the fact that very few platelets were found on the $200 \mathrm{~nm}$ alumina in contrast to the $20 \mathrm{~nm}$ membrane many microparticles were detected on this surface. Interestingly, all microparticles were found inside circular shaped areas of approximately $3 \mu \mathrm{m}$ in diameter. Since this is the approximate size of a platelet we speculate that this is evidence of transient, non-adherent platelet contact with the surface, which has triggered platelet microparticle generation. To the
\end{abstract}

\footnotetext{
N. Ferraz · J. Carlsson · M. K. Ott ( $₫)$

Department of Physical and Analytical Chemistry, Division of Surface Biotechnology, Uppsala University, BMC,

Husarg. 3, Box 577, Uppsala 751 23, Sweden

e-mail: Marjam.Ott@ytbioteknik.uu.se

\section{J. Hong}

Department of Oncology, Radiology and Clinical Immunology,

Division of Clinical Immunology, Rudbeck Laboratory, University Hospital, Uppsala University, Uppsala 75185 , Sweden
}

authors knowledge, this is the first study that demonstrates how nanotexture can influence platelet microparticle generation. The study highlights the importance of understanding molecular and cellular events on nano-level when designing new biomaterials.

\section{Introduction}

Blood-material contact leads to a series of interlinked events such as protein adsorption complement activation, platelet and leukocytes adhesion/activation and activation of the coagulation cascade. Platelets play a central role in the mechanism of thrombus formation. Activated platelets can regulate the subsequent behavior of other inflammatory cells such as monocytes and neutrophils [1]. Platelet agonists interact with specific receptors on the platelet plasma membrane and as a result the following physiological responses take place: release of platelet intracellular granule contents, drastic change in platelet shape that promotes platelet-platelet contact and adhesion, P-selectin expression on the platelet membrane, conformational change of the GPIIb/IIIa receptor leading to increased affinity towards soluble fibrinogen, rearrangement of the platelet membrane phospholipids converting it into a highly efficient procoagulant surface and platelet microparticle generation and release $[2,3]$. Platelet derived microparticles are submicroscopic $(0.1-1.0 \mu \mathrm{m})$ membrane vesicles released by activated platelets and have been described as excellent markers for platelet activation [4]. Platelets commonly bind to foreign material surfaces, which in many cases lead to platelet activation. Adsorbed fibrinogen has been described as the main protein involved in platelet adhesion to different classes of biomaterials [5, 
6]. However, other proteins such as von Willebrand factor (vWF), vitronectin and fibronectin are also involved in platelet adhesion and activation by artificial surfaces [7, 8]. It has been shown that the absence of platelet adhesion to a biomaterial surface does not prevent platelet activation. Therefore, other mechanisms must be involved in materialinduced platelet activation. Thrombin, ADP and complement components such as sC5b-9 and $\mathrm{C} 1 \mathrm{q}$ are presumed to be involved in such processes [3,9-11].

It is well known that surface topography of a biomaterial affects the biocompatibility in terms of cellular response and tissue integration. An exhaustive range of cells and their reactions to microtopography have been studied [12-14]. When looking specifically at platelets, it has been shown that surface topography in the micron range can modulate their activation, in terms of adhesion, generation of platelet microparticles (PMP) and P-selectin expression [15-17]. In recent years much attention has been paid to nanotopography when designing biologically inspired materials. Several authors have reviewed the interaction of various cell types with different nanoscale topographies $[12,18,19]$. However, few articles report on how nanotexture influences platelet behaviour [20, 21]. Hsu et al. has shown that by changing the nanotopography using diblock polymers one can influence platelet and monocyte activation. Dalby et al. however found no difference in platelet response between nano-islands and flat controls made from polymer demixing of polystyrene and poly 4-bromstyrene.

In the present work we have studied how anodized aluminium with different pore sizes (in the nanometer scale) modulates platelet adhesion and activation.

Aluminium has the advantage of easily being anodised into nano-porous alumina with different pore sizes [22]. The material in question has been evaluated as a potential bone implant coating [23] and as a stent coating for drug delivery [24]. In both cases showing favorable tissue compatibility, thus leading us to believe that it not only is suitable as a model substrate for investigating protein and cellular organization on nanoscale level, but may also be used as an implant material with a great potential for controlling type and magnitude of cellular and molecular events at the tissue-implant interface. We have compared alumina membranes with two pore sizes 20 and $200 \mathrm{~nm}$ in diameter. The membranes were incubated with whole blood and platelet rich plasma. Scanning electron microscopy was used to evaluate platelet morphological changes and microparticle generation. Immunocytochemical staining was also used to confirm platelet adhesion and activation by using antibodies against CD62P and CD41. To determine platelet activation in the fluid phase, platelet reduction and $\beta$-Thromboglobulin ( $\beta$-TG) release were evaluated.

\section{Materials and methods}

Anodisc $^{\mathrm{TM}}$ alumina membranes from Whatman International Ltd (Maidstone, England) were used in this work. The membranes are $25 \mathrm{~mm}$ in diameter and $60 \mu \mathrm{m}$ thick, with narrow pore size distribution. A 20 and $200 \mathrm{~nm}$ pore size alumina membranes were used in this study. Karlsson et al. [25] has previously characterized these materials and concluded that they have similar surface roughness and surface chemical characteristics.

\subsection{Heparin coating}

The slide chambers and the blood collection materials were heparin coated using the Corline method (Corline Systems AB, Uppsala, Sweden), following the manufacturer's recommendations.

\subsection{Whole blood experiments}

Whole blood from 10 healthy donors was collected in heparin-coated $50 \mathrm{ml} \mathrm{Falcon}{ }^{\circledR}$ tubes (Becton Dickinson, USA) containing soluble heparin (Bio Iberica, Barcelona, Spain) giving final concentrations of 0.25 and $0.5 \mathrm{IU}$ heparin $/ \mathrm{ml}$.

The slide chamber previously described by Hong et al. [26] was used for these experiments. The device is manufactured from polymethylacrylate (PMMA) and consists of two wells that can hold a maximum volume of $1.65 \mathrm{ml}$ each. After heparin coating each well was filled with $1.3 \mathrm{ml}$ of blood ( $1 \mathrm{ml}$ of blood was also collected in eppendorf tubes containing EDTA or citrate, these $0 \mathrm{~min}$ samples were later used as controls). The nano-porous alumina membranes were then placed covering the wells (as "lids"), thus two circular chambers were created. The slide chambers were rotated vertically at $22 \mathrm{rpm}$ for $60 \mathrm{~min}$ in a $37^{\circ} \mathrm{C}$ water bath. After incubation, $1 \mathrm{ml}$ of blood from each chamber was removed and mixed with EDTA- $\mathrm{K}_{3}$ or citrate, giving a final concentration of $4 \mathrm{mM}$ or $13 \mathrm{mM}$, respectively. The EDTA samples (including the 0 min controls) were analyzed for platelet numbers on a Coulter $\mathrm{A}^{\mathrm{c}} \mathrm{T}$ diff ${ }^{\mathrm{TM}}$ hematology analyzer (Coulter Corporation Miami, FL, USA). The citrated blood was centrifuged twice at $+4^{\circ} \mathrm{C}: 10 \mathrm{~min}$ at $1,000 \mathrm{~g}$ and $10 \mathrm{~min}$ at $10,000 \mathrm{~g}$. Thereafter the plasma samples were collected and stored at $-70^{\circ} \mathrm{C}$ for future analysis. The membranes were either fixed, dehydrated and critically point dried for scanning electron microscopy (SEM) studies or fixed for immunocytochemical staining.

\subsection{Platelet rich plasma experiments}

Blood from six healthy donors was collected in heparincoated $50 \mathrm{ml}$ Falcon ${ }^{\circledR}$ tubes (Becton Dickinson, USA) 
containing soluble heparin (Bio Iberica, Barcelona, Spain), giving a final concentration of $0.5 \mathrm{IU}$ heparin $/ \mathrm{ml}$. To obtain platelet rich plasma (PRP) blood was centrifuged at $190 \mathrm{~g}$, for $15 \mathrm{~min}$ at room temperature. Platelet count was read and the values ranged from 200 to $250 \times 10^{9} / 1$ for each experiment. The incubation and experimental preparation were conducted following the same protocol as used for whole blood.

\subsection{Detection of $\beta$-Thromboglobulin ( $\beta$-TG)}

Phosphate-buffered saline (PBS) containing 1\% (w/v) bovine serum albumin (Sigma-Aldrich, Inc. St Louis, USA) and 0.1\% TWEEN 20 (Sigma-Aldrich, Inc. St Louis, USA) was used as working buffer and PBS containing $0.1 \%$ TWEEN 20 and $0.02 \%$ Antifoam $^{\mathrm{TM}}$ (Pharmacia, Uppsala, Sweden) as washing buffer.

Citrate samples were analyzed for $\beta$-TG using Asserachrome $^{\text {TM }} \beta$-TG EIA kit (Diagnostica Stago, Asnieres-surSeine, France). Values are given in IU/ml.

\subsection{Scanning electron microscopy (SEM)}

After blood contact, the aluminium oxide membranes were washed with Hanks Balanced Salt Solution (HBSS) $\left(\mathrm{GIBCO}^{\mathrm{TM}}\right.$, Gran Island, NY, USA), fixed with $1.5 \%$ glutaraldehyde, dehydrated through a series of acetone concentrations $(25,50,70,80,90$ and $100 \%)$, critically point dried and sputtered with gold, finally to be studied using a LEO 1530, Gemini SEM.

The alumina membranes incubated with PRP were prepared for SEM analysis following the same protocol.

\subsection{Immunocytochemical staining}

The staining procedure was essentially done as described by Karlsson-Parra et al. [27]. The alumina membranes were washed with veronal buffered saline containing $0.75 \mathrm{mM} \mathrm{Ca}^{2+}$ and $2.5 \mathrm{mM} \mathrm{Mg}^{2+}$ (pH 7.4) and allowed to dry at room temperature. Fixation was done with ice cold $50 \%(\mathrm{v} / \mathrm{v})$ acetone for $30 \mathrm{~s}$, followed by $5 \mathrm{~min}$ in ice cold $100 \%$ acetone and allowed to dry at room temperature. The membranes were then incubated in PBS containing $1.8 \%$ (v/v) $\mathrm{H}_{2} \mathrm{O}_{2}$ for 15 min and thereafter blocked with human serum for $1 \mathrm{~h}$ at room temperature. Anti-CD62P and antiCD41 mouse immunoglobulins (Dako, Glostrup, Denmark) were used as primary antibodies, diluted 1/50 in PBS containing $4 \%$ BSA. The incubations were done in a humid chamber for $60 \mathrm{~min}$ at room temperature, followed by incubations with Envision ${ }^{\circledR}$ antimouse-HRP conjugated antibody for $60 \mathrm{~min}$ (Dako, Glostrup, Denmark). Finally, the membranes were stained in darkness for $15 \mathrm{~min}$, using 3-amino-9-ethyl carbazole reagent (AEC) (Dako, Glostrup, Denmark). Immuno-stained samples were examined using light microscopy (Nikon Eclipse E600 light microscope).

\subsection{Statistical analyses}

The results are expressed as mean \pm SE. Statistical significance was calculated with Student's $t$-test for unpaired samples, using Statview for Macintosh. $p$ values less than 0.05 were considered significant.

\section{Results}

\subsection{Platelet count}

Platelet counts were performed after whole blood and PRP incubations with the two alumina membranes. The results are expressed as a percentage of the values obtained for the 0 min controls (Fig. 1). A drop in platelet number was observed after whole blood contact with both alumina membranes, ranging from $11 \pm 2(0.25 \mathrm{IU}$ heparin/ml $)$ to $15 \pm 2 \%(0.5 \mathrm{IU}$ heparin/ml) for the $20 \mathrm{~nm}$ alumina and from $8 \pm 2(0.25 \mathrm{IU}$ heparin $/ \mathrm{ml})$ to $11 \pm 2 \%(0.5 \mathrm{IU}$ heparin/ml) for the $200 \mathrm{~nm}$ membrane. Thus showing a slightly higher platelet reduction for the $20 \mathrm{~nm}$ alumina. When measuring platelet count after incubation with PRP, we found no reduction in platelet numbers for either of the membranes.

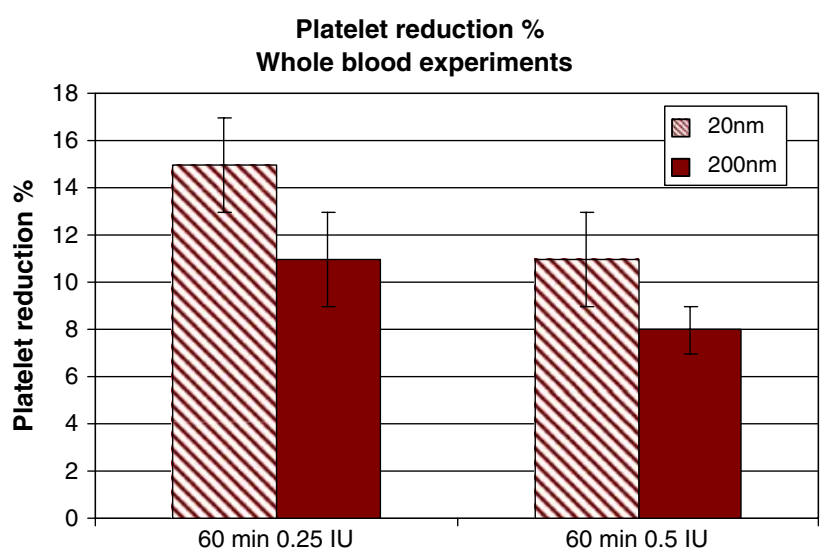

Fig. 1 Platelet counts performed after whole blood incubation with the two alumina membranes. The platelet reduction is expressed as a percentage of the values obtained for the $0 \mathrm{~min}$ controls. The data represents the mean $\pm \mathrm{SE}$ from experiments using blood from 10 different donors. When comparing the two membranes, higher platelet reduction is seen for the $20 \mathrm{~nm}$ compared to the $200 \mathrm{~nm}$ alumina, independent of heparin concentration. These values are not statistically significant although the same trend is observed in each of the 10 experiments performed 


\subsection{Detection of $\beta$-TG}

After whole blood incubation with the nano-porous alumina membranes, $\beta$-TG levels were significantly higher as compared to the 0 min control (Fig. 2), thus indicating platelet activation. However, no significant difference in $\beta$ TG levels between the two pore size membranes was seen.

\subsection{Scanning electron microscopy (SEM)}

Platelet number and morphology were evaluated by using SEM. Micrographs of whole blood experiments are shown

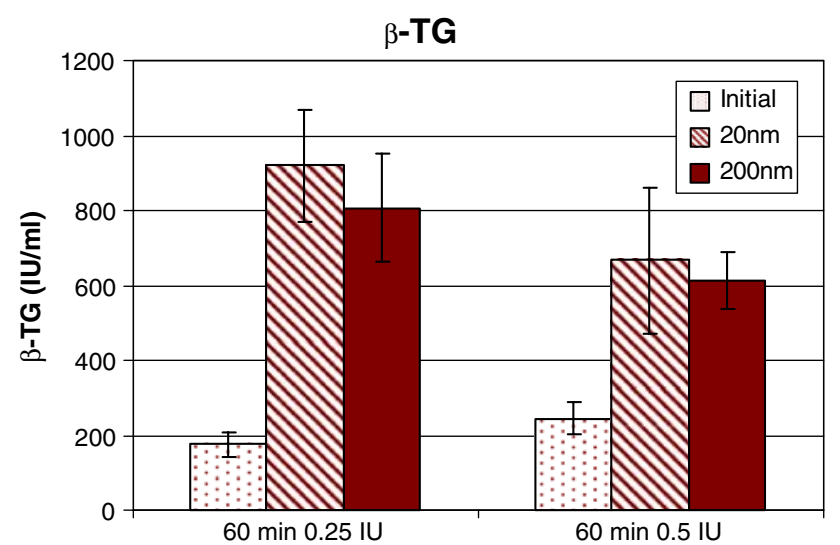

Fig. $2 \beta$-TG levels after whole blood incubation with the two alumina membranes. Initial values refer to $\beta$-TG levels in the $0 \mathrm{~min}$ controls. Data represents the mean $\pm \mathrm{SE}$ from experiments using blood from 10 different donors. $\beta$-TG values are significantly higher after whole blood contact with both pore size membranes $(p<0.05)$, thus indicating platelet activation upon contact with the biomaterials in Fig. 3. As can be seen in panels a and b, there is a clear difference between the two membranes. Specifically, very few platelets were found on the $200 \mathrm{~nm}$ alumina as compared to the $20 \mathrm{~nm}$ membrane. The platelets found on the $20 \mathrm{~nm}$ membrane showed typical signs of activation such as spread morphology and protruding filipodia. Despite the fact that very few platelets were found on the $200 \mathrm{~nm}$ alumina many microparticles could be seen on this surface. Interestingly, all microparticles were found inside circular shaped areas of approximately $3 \mu \mathrm{m}$ in diameter (see panels $b$ and $d$ ). Since this is the size of a platelet we assume that platelets have adhered and then detached leaving clusters of microparticles behind. This pattern is repeated on the entire $200 \mathrm{~nm}$ alumina surface. It should also be noted that between these circular shaped areas with microparticles the membrane is covered with what we assume to be cell debris from red blood cells, activated leukocytes and/or activated platelets.

The micrographs in Fig. 3 are representative for the experimental conditions: $60 \mathrm{~min}$ incubation and $0.5 \mathrm{IU} / \mathrm{ml}$ soluble heparin concentration. There was no significant difference in platelet appearance when changing the conditions to $0.25 \mathrm{IU}$ heparin/ml.

Alumina membranes were also incubated with platelet rich plasma (PRP) for $60 \mathrm{~min}(0.5 \mathrm{IU}$ heparin/ml) and thereafter observed under the SEM. Results are shown in Fig. 4. While the $200 \mathrm{~nm}$ membrane has microparticles aggregates distributed on the entire surface (panels $b$ and $d$ ) the $20 \mathrm{~nm}$ membrane is practically "clean" with the exception of traces of cell debris (panels a and c). No adhering platelets on either of the material surfaces were found. This result is in agreement with the platelet count.
Fig. 3 SEM micrographs after incubation with whole blood. For the $20 \mathrm{~nm}$ membranes (Panels a and c) quite a large number of platelets are detected which show typical signs of activation such as spread morphology and protruding filipodia. No microparticles are seen on this surface. On the $200 \mathrm{~nm}$ membranes (Panels b and d) many microparticles can be seen but no platelets. Interestingly, all microparticles are found inside circular shaped areas of approximately $3 \mu \mathrm{m}$ in diameter. Since this is the size of a platelet we assume that platelets have adhered and then detached leaving clusters of microparticles behind
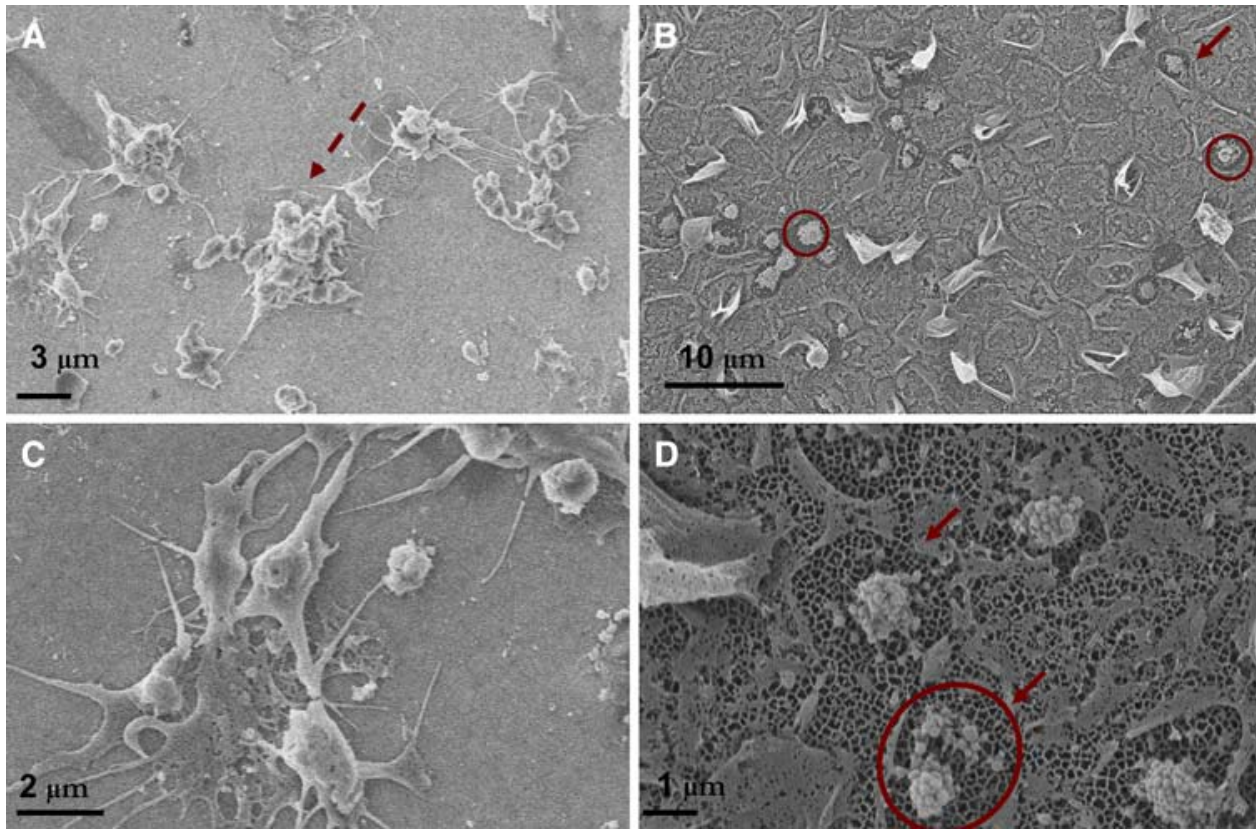
Fig. 4 SEM micrographs after incubation with platelet rich plasma. No platelets are detected on the $20 \mathrm{~nm}$ alumina (Panels a and c). On the $200 \mathrm{~nm}$ membrane (Panels $\mathbf{b}$ and $\mathbf{d}$ ) clusters of PMPs can be seen. This membrane does however look different than when incubated with whole blood (see Fig. 3) leading us to believe that the cell debris seen on the whole blood samples is caused by red blood cells and/or activated leukocytes
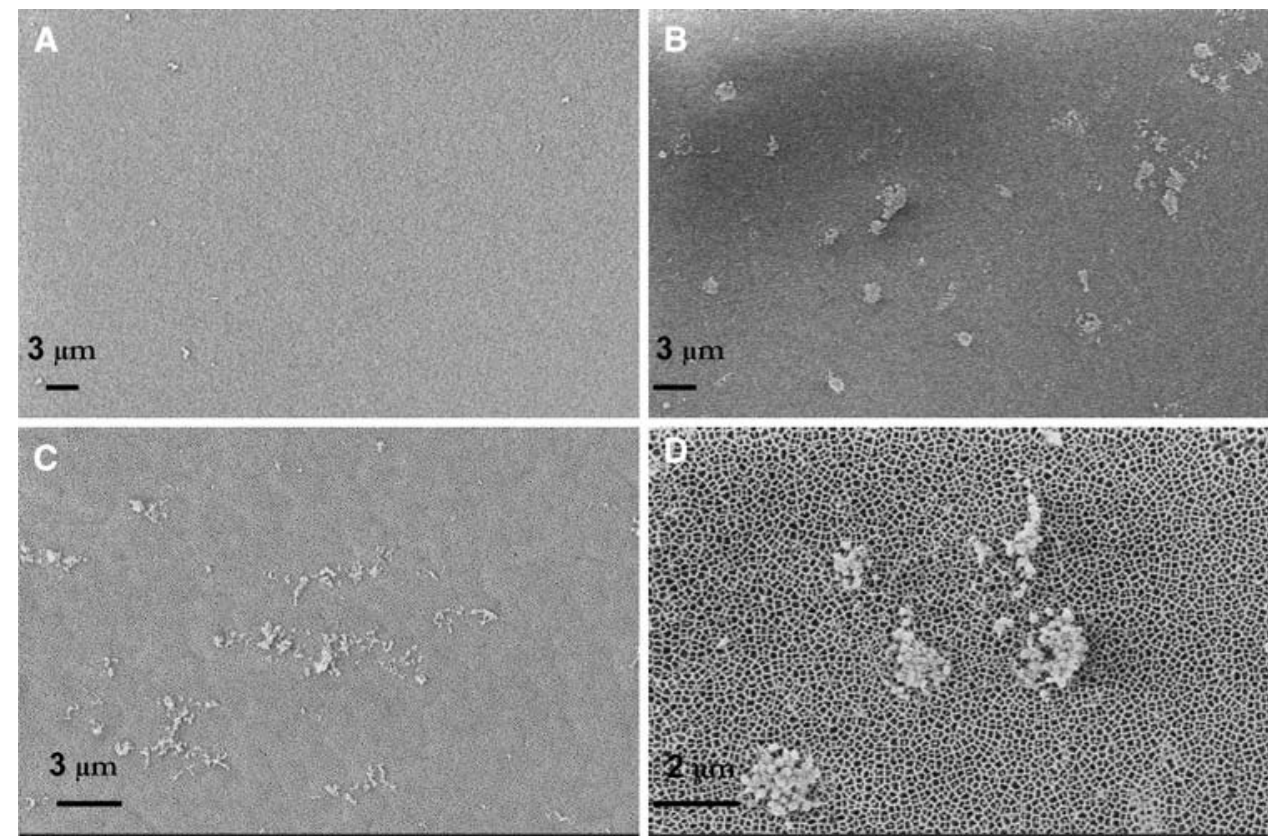

When comparing Figs. 3 and 4 the magnitude of erythrocyte and/or leukocyte influence on platelet interaction with nano-porous alumina is evident. Also when looking at the results of 20 versus $200 \mathrm{~nm}$ alumina it is clear that nanotexture has a huge effect on platelet adhesion and activation.

\subsection{Immunocytochemical staining}

In addition to the SEM study bound platelets were analyzed by light microscopy using specific antibodies that were visualized by means of immunocytochemical staining. Antibodies against CD41 (glycoprotein IIb of the GPIIb/ IIIa complex) were used to identify surface bound platelets and antibodies against CD62P (P-selectin) were used to detect activated platelets. It should be noted that platelet microparticles (PMP) expose GPIIB/IIIa complex and can also express activation markers as P-selectin. Figure 5 shows representative pictures of alumina membranes after $60 \mathrm{~min}$ of incubation with whole blood ( $0.5 \mathrm{IU}$ soluble heparin/ml). CD41 and CD62P positive events are present
Fig. 5 Light microscopy pictures from immunocytochemical staining of alumina membranes after incubation with whole blood. Upper panels (a and b) show positive events for CD41. Lower panels (c and d) show positive events for CD62P. Taken together with the SEM results (see Fig. 3) the positive events detected on the $20 \mathrm{~nm}$ membranes (panels a and c) could be described as activated platelets while what is observed on panels b and d (200 nm alumina) most likely represent clusters of microparticles most of which express P-selectin (panel d). Scale bars represent $9 \mu \mathrm{m}$
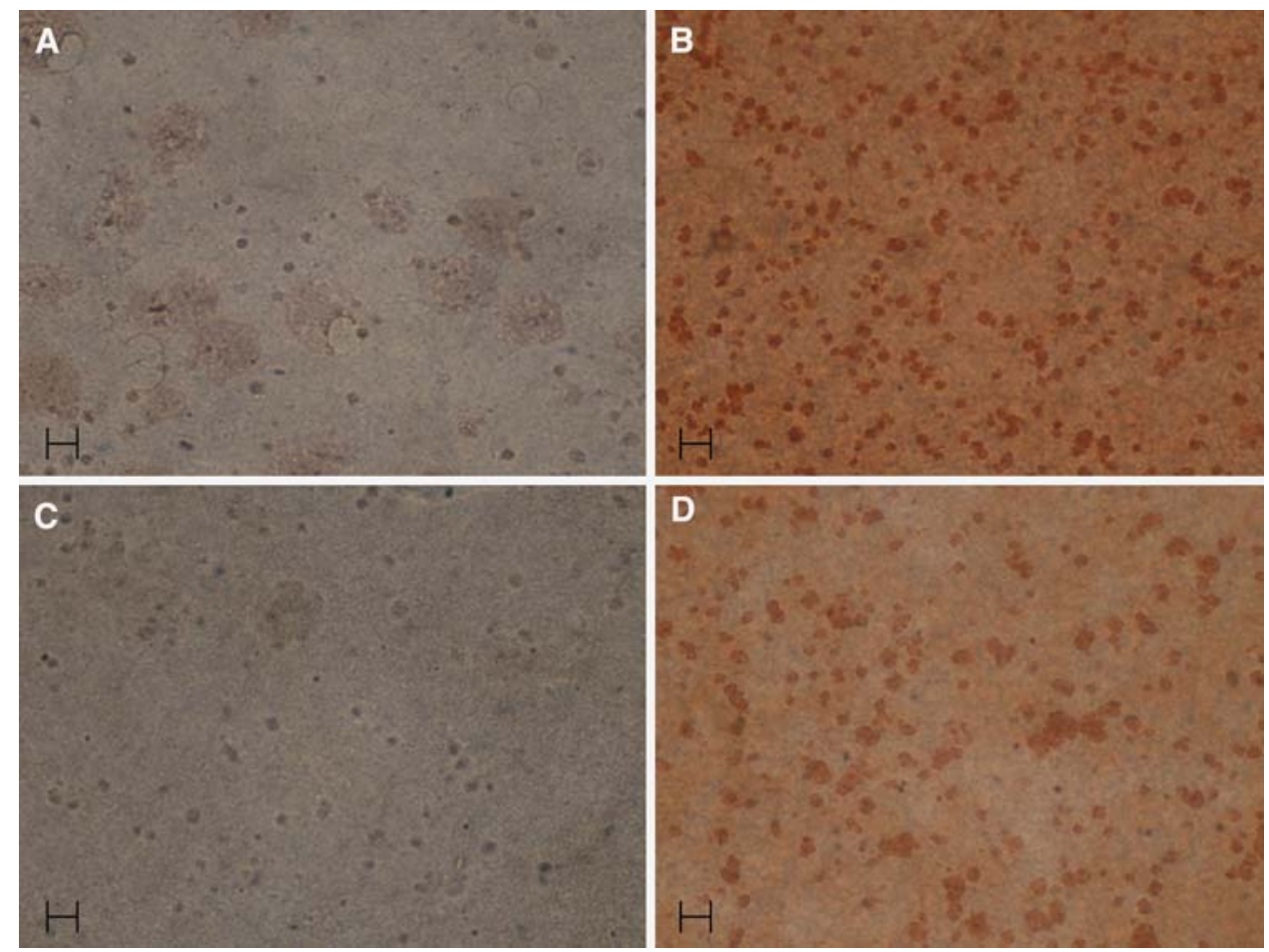
at higher levels on the $200 \mathrm{~nm}$ alumina compared to the $20 \mathrm{~nm}$ membrane. The size of PMPs range from 0.1 to $0.3 \mu \mathrm{m}$ in diameter and when aggregating each cluster could have a diameter of 1-3 $\mu \mathrm{m}$. Taking this into consideration together with the SEM study we presume that what we see on the $200 \mathrm{~nm}$ alumina surface are aggregates/ clusters of PMPs that express P-selectin. The positive events seen on the $20 \mathrm{~nm}$ membranes are however most likely activated platelets since no PMPs are found on this surface. A comparison between panels a and c illustrates that most of the platelets bound to the $20 \mathrm{~nm}$ alumina are activated. This result correlates well with the SEM observation of the morphology of the platelets.

\section{Discussion}

The slide chamber model used in the present work makes it possible to analyse both the biomaterial surface in terms of protein and cell adhesion and the cellular and molecular events that take place in the fluid phase. We combined two microscopy techniques to examine platelet interactions with nano-porous alumina. Additionally, we determined the change in platelet number in the fluid phase after whole blood and PRP contact with the studied materials. $\beta$-TG release was also studied after whole blood contact with the alumina membranes.

Taken together the immunocytochemical staining and SEM analysis of the nano-porous alumina membranes indicate that many activated platelets but no microparticles were detected on the $20 \mathrm{~nm}$ membrane. On the contrary, $200 \mathrm{~nm}$ alumina promotes generation of P-selectin expressing PMPs. However platelets could rarely be found on this surface. Godo et al. [28] has reported high platelet reactivity together with low platelet adhesion to polyvinyl alcohol hydrogels. By using fluorescent-video microscopy they detect transient, non-adherent platelet contacts with the material. They hypothesized that after contact with a layer of adsorbed proteins on a biomaterial surface, platelets can either adhere or "bounce off". Moreover, the amount and conformation of adsorbed proteins will probably influence the number of platelets that are activated by the transient contact. It makes sense to believe that the circular shaped areas of approximately $3 \mu \mathrm{m}$ in diameter identified on the $200 \mathrm{~nm}$ alumina membranes (see circles in Fig. 3) are evidences of platelet transient, non-adherent contacts which seem to have triggered PMP release. It should also be noted that between the circular shaped areas with microparticles the membrane is covered with what we assume to be cell debris from activated platelets, red blood cells and/or activated leukocytes. This reasoning comes from the fact that when incubating the $200 \mathrm{~nm}$ alumina with platelet rich plasma (where red blood cells and leukocytes have been removed) the membrane is "clean" except for the clusters of microparticles found throughout the surface (see Fig. 4). When incubating the $20 \mathrm{~nm}$ membrane with platelet rich plasma no platelets were detected. This is likely due to the fact that red blood cells and/or leukocytes have an effect on platelet adhesion to the alumina membrane. Several authors have investigated the role of red blood cells in platelet activation and adhesion. Alkhamis and coworkers established that red blood cells release a significant fraction of ADP which is enough to induce platelet aggregation and adhesion [29]. Valles et al. [30] demonstrated that interactions between red blood cells and platelets amplify platelet reactivity. Our results clearly show the importance of working with whole blood when evaluating platelet interactions with artificial surfaces.

When looking at platelet number in the fluid phase, we found that there was a higher reduction after whole blood contact with $20 \mathrm{~nm}$ alumina than with the $200 \mathrm{~nm}$ membrane. However, the difference between the two alumina membranes is not as significant as expected when looking at the SEM micrographs. Gemmell [31] has pointed out that given the large number of microparticles that potentially shed from a single platelet it is not conceivable that microparticle generation would appreciably lower bulk platelet count. However Nomura and Fukuhara [32] state that it is unclear whether PMPs arise from complete conversion of a few platelets or from partial conversion of many platelets. Considering that no platelets were seen on the $200 \mathrm{~nm}$ membrane, we speculate that the numerous PMPs generated after whole blood contact with this membrane might have caused some platelets to disintegrate after deposition of the microparticles thus causing a slight decrease in platelet numbers in the bulk.

Concerning $\beta$-TG release no difference between the membranes was detected. A significant increase in $\beta$-TG levels was however observed for both membranes independent of poresize. These results are not surprising since platelet activation was indeed seen on both membranes but reflected in different ways; microparticle release was seen on the $200 \mathrm{~nm}$ membrane while adhering platelets with activated morphology were observed on the $20 \mathrm{~nm}$ membrane.

In summary, platelet activation by the two pore size alumina membranes showed different characteristics as reflected by platelet and microparticle adhesion. Several factors should be considered when looking at these results, e.g. the influence of complement activation. The complement system has been reported to promote platelet activation in several ways. C1q is presumed to be involved in GPIIb/IIIa activation and P-selectin expression [11]. Insertion of C5b-9 on platelet membranes has also been described as a possible mechanisms leading to PMP generation [10]. In our previous work we found that $200 \mathrm{~nm}$ 
alumina membranes cause higher complement activation than $20 \mathrm{~nm}$ membranes [33]. Therefore, we speculate that the difference seen in platelet adhesion, activation and PMP generation in this study is influenced by the difference in complement activation between the membranes. Since there is no difference in surface chemistry or topography between the two alumina membranes [25] we conclude that the results obtained in this study are a consequence of the pore size. The different pore diameters result in a difference in accessible surface area for the proteins depending on their size and shape. Hence different proteins patterns are most likely created on the two membranes. This will in turn influence the availability of specific receptors and binding sites, thus affecting platelet interaction with the biomaterial surface.

\section{Conclusion}

The present study shows that a small difference in nanopore size has a huge effect on platelet adhesion and activation. Numerous articles report on how different biomaterials influence platelet microparticle generation $[31,34]$. However, to the author's knowledge, this is the first study that shows how nanotexture can affect PMP generation. We expect that better understanding of molecular and cellular events on nano-level will play a significant role when designing new biomaterials.

Acknowledgments The authors would like to thanks Leif Ljung at the Department of Medical Cell Biology for technical assistance with the SEM studies. We also wish to thank all the people who kindly donated blood for these experiments.

\section{References}

1. A.J. Marcus, in "Inflammation: Basic Principles and Clinical Correlates" (Lippincott Williams \& Wilkins, Philadelphia, 1999), p. 77

2. B.A. Bouchard, S. Butenas, K.G. Mann, P.B. Tracy, in "Platelets" (Academic Press, San Diego, 2002), p. 229

3. M.B. Gorbet, M.V. Sefton, Biomaterials 25, 5681 (2004)

4. L.L. Horstman, Y.S. Ahn, Crit. Rev. Oncol. Hematol. 30, 111 (1999)
5. M. Broberg, C. Erikksson, H. Nygren, J. Lab. Clin. Med. 139, 163 (2002)

6. W.B. Tsai, J.M. Grunkemeier, C.D. McFarland, T.A. Horbett, J. Biomed. Mater. Res. 60, 348 (2002)

7. J.M. Grunkemeier, W.B. Tsai, C.D. McFarland, T.A. Horbett, Biomaterials 21, 2243 (2000)

8. M. Broberg, H. Nygren, Colloids Surf B: Biointerfaces 11, 67 (1998)

9. P.J. Sims, T. Wiedmer, Immunol. Today 12, 338 (1991)

10. P.J. Sims, T. Wiedmer, Semin. Cell Biol. 6, 275 (1995)

11. E.I.B. Peerschke, K.B.M. Reid, B. Ghebrehiwet, J. Exp. Med. 178, 579 (1993)

12. R.G. Flemming, C.J. Murphy, G.A. Abrams, S.L. Goodman, P.F. Nealey, Biomaterials 20, 573 (1999)

13. C. Eriksson, J. Lausmaa, H. Nygren, Biomaterials 22, 1987 (2001)

14. A. Curtis, C. Wilkinson, Biomaterials 18, 573 (1997)

15. J.Y. Park, C.H. Gemmell, J.E. Davies, Biomaterials 21, 2671 (2001)

16. L. Kikuchi, J.Y. Park, C. Victor, J.E. Davies, Biomaterials 26, 5285 (2005)

17. K.R. Milner, A.J. Snyder, C.A. Siedlecki, J. Biomed. Mater. Res. 76A, 561 (2006)

18. A. Curtis, C. Wilkinson, Trends Biotechnol. 19, 97 (2001)

19. E.K.F. Yim, K.W. Leong, Nanomedicine: Nanotechnol. Biol. Med. 1, 10 (2005)

20. S.H. Hsu, C.M. Tang, C.C. Lin, Biomaterials 25, 5593 (2004)

21. M.J. Dalby, G.E. Marshall, H.J.H. Johnstone, S. Affrossman, M.O. Riehle, IEEE Trans. Nanobiosci. 1, 18 (2002)

22. G.E. Thompson, Thin Solid Films 297, 192 (1997)

23. M. Karlsson, E. Pålsgård, P.R. Wilshaw, L. Di Silvio, Biomaterials 24, 3039 (2003)

24. H. Wieneke, O. Dirsch, T. Sawitowski, Y.L. Gu, H. Brauer, U. Dahmen, A. Fischer, S. Wnendt, R. Erbel, Catheter. Cardiovasc. Interv. 60, 399 (2003)

25. M. Karlsson, A. Johansson, L. Tang, M. Boman, Microsc. Res. Tech. 63, 259 (2004)

26. J. Hong, K. Nilsson Ekdahl, H. Reynolds, R. Larsson, B. Nilsson, Biomaterials 20, 603 (1999)

27. A. Karlsson-Parra, U. Forsum, L. Klareskog, O. Sjoberg, J. Immunol. Methods 64, 85 (1983)

28. M.N. Godo, M.V. Sefton, Biomaterials 20, 1117 (1999)

29. T.M. Alkhamis, R.L. Beissinger, J.R. Chediak, ASAIO Trans. 34, 868 (1988)

30. J. Valles, M.T. Santos, J. Aznar, A.J. Marcus, V. Martinez-Sales, M. Portoles, M.J. Broekman, L.B. Safier, Blood 78, 154 (1991)

31. G.H. Gemmel, J. Biomater. Sci. Polym. Ed. 12, 933 (2001)

32. S. Nomura, S. Fukuhara, in "Platelets and Megakaryocytes, Methods in Molecular Biology" (Humana Press Inc, Totawa, NJ, 2004), p. 260

33. N. Ferraz, B. Nilsson, J. Hong, M. Karlsson-Ott, J. Biomed. Mater. Res. A. (2008). doi:10.1002/jbm.a.31818

34. C.A. Siedlecki, I.W. Wang, J.M. Higashi, K. Kottke-Marchant, R.E. Marchant, Biomaterials 20, 1521 (1999) 